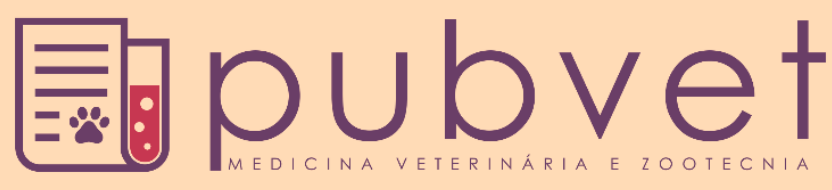

https://doi.org/10.31533/pubvet.v14n10a678.1-6

\title{
Correção cirúrgica de tórax paradoxal devido a traumatismo por mordedura em um cão: Relato de caso
}

\author{
Tiago Trindade Dias ${ }^{1 *}$, Mariana Wilhelm Magnabosco ${ }^{2} \bullet$, Péter de Lima Wachholz ${ }^{3}$, Tainá \\ Ança Evaristo $^{4}$, Thaís Cozza dos Santos ${ }^{\circ}{ }^{\circ}$, Yohana Fernanda Henz ${ }^{6}{ }^{\circ}$, Vitória Ramos de \\ Freitas $^{7}{ }^{\circ}$, Beatriz Persici Maroneze ${ }^{8} \bullet$, Paula Priscila Correia Costa ${ }^{\circ}$, Amanda Leal de \\ Vasconcellos $^{10} 0$ \\ ${ }^{I}$ Médico Veterinário graduado pela Universidade da Região da Campanha. Bagé - RS, Brasil. \\ ${ }^{2}$ Médica Veterinária Residente em Diagnóstico por Imagem pela Universidade Federal de Pelotas. Pelotas (UFPel)-RS, Brasil. \\ ${ }^{3}$ Médico Veterinário Residente em Clínica Médica de Pequenos Animais pela UFPel. Pelotas - RS, Brasil. \\ ${ }^{4}$ Graduanda em Medicina Veterinária pela UFPel. Pelotas - RS, Brasil \\ ${ }^{5}$ Médica Veterinária Mestranda pela UFPel. Pelotas - RS, Brasil. \\ ${ }^{6}$ Médica Veterinária pela Universidade Federal de Santa Catarina. Curitibanos - SC, Brasil. \\ ${ }^{7}$ Médica Veterinária Residente em Anestesiologia Veterinária pela UFPel. Pelotas - RS, Brasil. \\ ${ }^{8}$ Médica Veterinária Mestranda pela Universidade Federal do Rio Grande do Sul. Porto Alegre - RS, Brasil. \\ ${ }^{9}$ Docente no Departamento de Clínicas Veterinárias (DCV), Hospital de Clínicas Veterinárias (HCV/UFPel),UFPel \\ ${ }^{10}$ Docente no Departamento de Clínica Médica de Pequenos Animais (UECE), Doutora em Ciências Veterinárias (Unesp) \\ *Autor para correspondência, E-mail: tiagotdias@hotmail.com
}

Resumo. O traumatismo torácico está entre as afecções mais frequentes na emergência de pequenos animais, podendo se apresentar com diferentes níveis de gravidade. Os achados mais comuns são pneumotórax, hemotórax, fraturas de costelas e hérnias diafragmáticas. Nem sempre essas lesões necessitam de procedimentos cirúrgicos, porém quando o grau de acometimento é elevado, a exploração cirúrgica é aceitável para avaliar a real extensão dos ferimentos internos. O presente trabalho tem o objetivo de relatar um caso de um cão atacado na região torácica, descrevendo sobre a abordagem do paciente politraumatizado, além do diagnóstico, tratamento e cuidados no pós-operatório. O paciente foi atendido no Hospital Veterinário Dra. Renata Saccaro e no momento da consulta apresentava extensas lesões de tecidos moles, pneumotórax e fraturas de costela, resultando na perda da estabilidade do tórax. Após os primeiros atendimentos e exames complementares optou-se por encaminhar ao bloco cirúrgico, onde realizou-se a inspeção dos órgãos torácicos, seguido por toracorrafia com colocação de dreno torácico. O paciente recebeu cuidados intensivos até a sua alta médica e continuou sendo acompanhado até sua completa recuperação. Este trabalho demonstra a importância da agilidade do médico veterinário em diagnosticar as comorbidades que acompanham o paciente politraumatizado para, desta forma, encaminhá-lo para o tratamento efetivo o mais rápido possível e salvar o paciente.

Palavras chave: cirurgia, tórax, trauma, pequenos animais

\section{Surgery correction of paradoxical thorax wall due to bite trauma in a dog: Case report}

Abstract. Thoracic trauma is among the most common conditions in small animals and can be presented with different levels of severity. The most common findings are pneumothorax, hemothorax, rib fractures and diaphragmatic hernias. These injuries do not always require surgical procedures, but when the degree of involvement is high, surgical exploration is acceptable to evaluate the real extent of internal injuries. The present work aims to report a case of a dog attacked in the thoracic region, describing an approach to the multiple trauma patient, in addition to diagnosis, treatment and care in the postoperative 
period. The patient was attended at Hospital Veterinário Renata Saccaro and had extensive soft tissue injuries, pneumothorax and rib fractures, resulting in loss of chest stability. After the first medical appointments and complementary exams, the patient was referred to the operating room, where the inspection of the thoracic organs was performed, followed by thoracorraphy with placement of a chest tube. The patient received intensive care until medical release and continued to follow up until his complete recovery. This paper demonstrates the importance of the veterinarian's agility in diagnosing the comorbidities that accompany the polytrauma patient in order to refer him to effective treatment as soon as possible and save the patient.

Keywords: surgery, thorax, trauma, small animals

\section{Corrección quirúrgica de tórax paradoxal debido a traumatismo por mordedura en un perro: Reporte de caso}

Resumen. El traumatismo torácico está entre las afecciones más frecuentes en la emergencia de pequeños animales, pudiéndose presentar con diferentes grados de severidad. Los hallazgos más comunes son neumotórax, hemotórax, fracturas de costillas y hernias diafragmáticas. Muchas veces estas lesiones no necesitan de procedimientos quirúrgicos, sin embargo, cuando el grado de acometimiento es alto, la exploración quirúrgica es aceptable para evaluar la real extensión de las heridas internas. El presente trabajo tiene como objetivo relatar el caso de un perro atacado en región torácica, describiendo sobre el abordaje del paciente politraumatizado, además del diagnóstico, tratamiento y cuidados en el post-quirúrgico. El paciente fue atendido en el Hospital Veterinário Dra. Renata Saccaro y en el momento de la consulta presentaba extensas heridas en los tejidos blandos, neumotórax y fracturas de costilla, resultando en la pérdida de la estabilidad torácica. Luego de los primeros atendimientos y exámenes complementares se optó por encaminar a la sala de cirugía, en donde se realizó la inspección de los órganos torácicos, seguido de toracorrafia con colocación de dreno torácico. El paciente recibió cuidados intensivos hasta su alta médica y continuó siendo acompañado hasta su completa recuperación. Este trabajo muestra la importancia de la agilidad del médico veterinario en diagnosticar las comorbilidades que acompañan al paciente politraumatizado para, de esta forma, encaminarlo para el tratamiento efectivo lo más rápido posible y salvar al paciente.

Palabras clave: cirugía, tórax, trauma, pequeños animales

\section{Introdução}

Durante a rotina emergencial de pequenos animais, o traumatismo torácico está frequentemente presente, com maior prevalência em caninos do que em felinos domésticos (Olsen et al., 2002). As lesões ocasionadas por estes traumas podem ser secundárias ao impacto contuso ou penetrantes (Fossum, 2014; Nelson \& Couto, 2015).

Os achados clínicos provenientes desta afecção incluem pneumotórax, contusões pulmonares, fraturas de costela, tendo consequência a ocorrência de hérnias diafragmáticas e hemotórax (Aguiar, 2011).

As fraturas de costela é um dos achados mais frequentes, podendo ocasionar uma instabilidade torácica que faz com que o segmento fraturado se mova paradoxalmente com os movimentos respiratórios, resultando no acúmulo e recaptação de $\mathrm{CO}_{2}$, pela pressão e inabilidade de inflar e desinflar, exercido sob o(s) lobo(s) pulmonares afetados (Aguiar, 2011; Fossum, 2014).

Outro achado bastante comum é o pneumotórax, que pode ser classificado como aberto ou fechado. Quando ocasionado por uma lesão penetrante no pulmão, que permite o extravasamento de ar no tórax, classifica-se como pneumotórax fechado. Quando ocorre uma lesão na parede torácica, que possibilita a entrada de ar atmosférico para dentro da cavidade pleural, classifica-se como pneumotórax aberto (Aguiar, 2011; Fossum, 2014). 
A radiografia e a tomografia computadorizada são os exames complementares de eleição para auxiliar no diagnóstico, trazendo uma melhor análise e localização da extensão das lesões torácicas (Fossum, 2014; Nelson \& Couto, 2015; Slatter, 2007). As primeiras condutas para o tratamento do paciente com trauma torácico seguem os princípios do protocolo ABCDE de atendimento emergencial, onde os sinais clínicos serão avaliados para cada caso, seguido de estabilização do paciente e, por fim, optar pela melhor conduta a ser tomada clinicamente ou, se houver a necessidade, por abordagem cirúrgica para a correção do defeito (Bastos et al., 2008).

O presente trabalho visa relatar o caso de um traumatismo torácico em um cão que gerou tórax paradoxal, abordando sobre sinais clínicos, diagnóstico, técnica cirúrgica e tratamento pós-operatório do paciente.

\section{Material e métodos}

Foi atendido no Hospital Veterinário Dra. Renata Saccaro, localizado em Caxias do Sul - RS, um cão, macho, sem raça definida (SRD), de sete anos de idade, pesando $10 \mathrm{~kg}$, com histórico de, no momento do passeio, ter sofrido um ataque por outro cão. Foi mencionado também, que o paciente apresentou muito sangramento pelas lesões dos ferimentos.

Ao exame físico, observou-se várias lesões de pele na região lateral direita do tórax. No local de lesão torácica, foi introduzida uma sonda uretral $\mathrm{n}^{\circ} 8$ para avaliar a dimensão das lacerações, constatando-se que havia comunicação entre elas. O paciente também apresentava enfisema subcutâneo e dispneia, com angústia respiratória.

Para estabilização do paciente, realizou-se oxigenoterapia com oxigênio $100 \%$ fornecido por máscara, toracocentese para restabelecer a pressão negativa intratorácica, fluidoterapia com solução de ringer simples em taxa de $90 \mathrm{ml} / \mathrm{kg} / \mathrm{hora}$, objetivando corrigir desequilíbrios hidroeletrolíticos e, administração de metadona $(0,3 \mathrm{mg} / \mathrm{kg})$ via intramuscular, para analgesia.

Após a estabilização, foi realizada a radiografia torácica em projeções ortogonais para melhor avaliar as lesões. Constatou-se moderada presença de ar em subcutâneo lateral direito do tórax, aumento da radioluscência da cavidade torácica, associada a intenso padrão alveolar do lobo pulmonar caudal esquerdo e, discreto desvio cardíaco para a esquerda. Presença de descontinuidade óssea em corpo de nona, décima e décima-primeira costelas também foi constatado. As alterações encontradas foram compatíveis com enfisema subcutâneo e fratura de nona, décima e décima primeira costelas, associadas a pneumotórax e consolidação pulmonar de lobo caudal esquerdo (Figura 1).

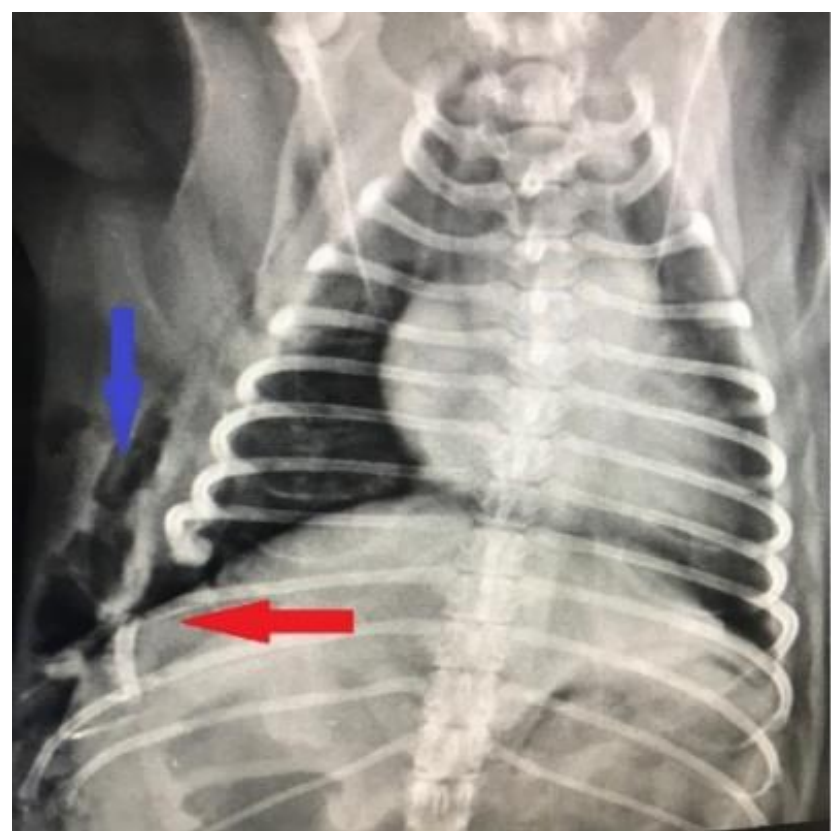

Figura 1. Radiografia em projeção ventrodorsal de tórax evidenciando enfisema subcutâneo (seta azul), fratura de nona (seta vermelha), décima e décima-primeira costelas, pneumotórax e consolidação pulmonar de lobo caudal esquerdo. 
Foi indicado a realização de exames hematológicos de emergência para o encaminhamento do paciente para procedimento cirúrgico, com o objetivo de reparar a instabilidade da parede torácica.

Realizou-se medicação pré-anestésica (MPA) com metadona $(0,3 \mathrm{mg} / \mathrm{kg})$ e midazolam $(0,3 \mathrm{mg} / \mathrm{kg})$, via intramuscular. No pré-cirúrgico, foi realizada a indução anestésica endovenosa com propofol $(4 \mathrm{mg} / \mathrm{kg})$, seguida da realização de ampla tricotomia da região lateral direita do tórax, com o paciente posicionado em decúbito lateral esquerdo e, por fim, antissepsia da região. Na manutenção anestésica, manteve-se o paciente com anestesia inalatória, utilizando-se isoflurano ao efeito, bem como a monitoração constantes dos parâmetros cardiorrespiratórios. A técnica cirúrgica consistiu em ampla incisão no nono espaço intercostal, observando-se grande dilaceração da musculatura com lesões penetrantes que comunicavam o tórax com o meio externo (Figura 2).

O tecido desvitalizado foi excisionado, verificando-se a viabilidade dos órgãos internos. Realizou-se o "teste do borracheiro", que consiste na administração de solução salina morna para dentro do tórax, com a finalidade de observar a aerostasia pulmonar. Na sequência, foi introduzido um dreno torácico, para remover o ar residual da cavidade pleural, com o objetivo de restabelecer a pressão intratorácica. E, por fim, realizou-se aproximação das costelas, com a colocação prévia de sutura de náilon 2-0, seguida por rafia da musculatura torácica com um padrão de sutura Sultan, redução do espaço morto e dermorrafia com um padrão de sutura Wolff, ambas com náilon 3-0 (Figura 3).

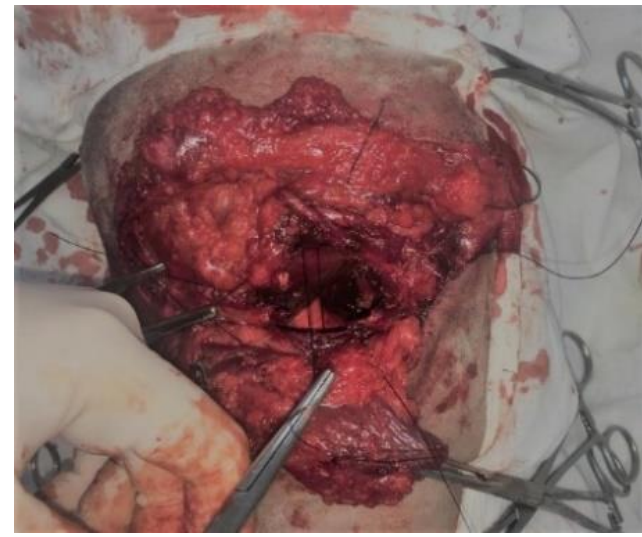

Figura 2. Dilaceração grave de musculatura costal direita

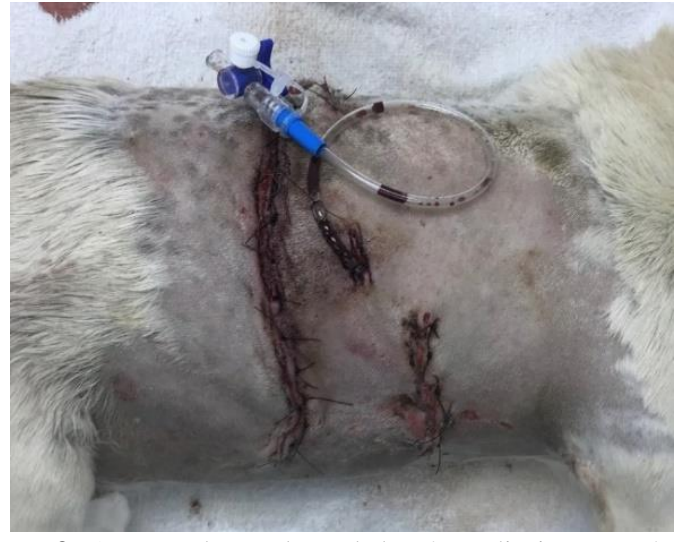

Figura 3. Aspecto latero-lateral do tórax direito em pósoperatório imediato

No pós-operatório, o paciente ficou sob cuidados intensivos, por um período de 72 horas sendo administrado antibioticoterapia com cefalotina, (20 mg/kg/via intravenosa/a cada 8 horas), antiinflamatório (meloxican, $0,1 \mathrm{mg} / \mathrm{kg} / \mathrm{via}$ subcutânea/a cada 24 horas), analgésico opioide (metadona, 0,3 $\mathrm{mg} / \mathrm{kg} / \mathrm{via}$ subcutânea/cada 8 horas) e, protetor gástrico (omeprazol, $0,7 \mathrm{mg} / \mathrm{kg} / \mathrm{via}$ intravenosa/cada 24 horas). Também foi realizada drenagem diária do pneumotórax residual. O dreno foi retirado somente quando se obteve uma drenagem inferior a 20-30 ml de conteúdo dentro de 24 horas.

O paciente recebeu alta médica com prescrição terapêutica a domicílio de: cefalexina $(20 \mathrm{mg} / \mathrm{kg} / \mathrm{via}$ oral/cada 12 horas) por 10 dias, carprofeno $(4,4 \mathrm{mg} / \mathrm{kg} / \mathrm{via}$ oral $/ 24-24 \mathrm{~h}$ ) por 10 dias, tramadol (3 $\mathrm{mg} / \mathrm{kg} / \mathrm{via}$ oral $/ 8-8 \mathrm{~h})$ por 4 dias, dipirona $(25 \mathrm{mg} / \mathrm{kg} / \mathrm{via}$ oral $/ 12-12 \mathrm{~h})$ por 4 dias e omeprazol (1 $\mathrm{mg} / \mathrm{kg} /$ via oral/24-24h) por 7 dias. Foi recomendado também, manter o paciente em repouso, realizar limpeza dos pontos cirúrgicos duas vezes ao dia com solução fisiológica, uso de colar elisabetano e/ou roupa cirúrgica e retornar a cada cinco dias para reavaliação e realização de inspeção da ferida até seu completo fechamento.

\section{Discussão}

No presente relato, o paciente acometido era um cão, macho, SRD. Olsen et al. (2002) citam que cães estão mais susceptíveis a traumatismos torácicos por brigas do que gatos, corroborando com os fatos do caso relatado. Os achados clínicos apresentados pelo paciente foram: enfisema subcutâneo, fraturas de costela e pneumotórax, concordando com os achados citados por Aguiar (2011) para traumatismo torácico proveniente de brigas entre cães. O paciente ainda apresentava instabilidade da 
parede torácica, fazendo com que o segmento fraturado se movimentasse paradoxalmente com os movimentos respiratórios. Fossum (2014) afirma que para o movimento paradoxal acontecer, deve-se ter uma ou mais costelas nos dois lados do ponto de impacto fraturadas. As costelas nona, décima e décima primeira do cão deste trabalhado estavam fraturadas gerando a instabilidade.

Para estabilizar o paciente, que apresentava dispneia devido ao trauma, foi administrado oxigeno terapia seguida de toracocentese no sétimo espaço intercostal. Johnson et al. (2013) e Tobias \& Johnston (2013) mencionam que estas medidas devem ser tomadas primeiramente ao se abordar pacientes acometidos de trauma torácico.

Truitt et al. (2011) ainda mencionam que para fornecer um maior conforto para o paciente, colocase ele em decúbito lateral sobre o lado afetado, estabilizando a parede torácica e assim controlando a dor para melhorar a ventilação pulmonar.

O que levou a confirmação do diagnóstico de fraturas de costela e instabilidade torácica, foi a realização de exame físico e radiografia torácica. Johnson et al. (2013) e Tobias \& Johnston (2013) citam que para ter a certeza destes achados, deve-se lançar mão de um minucioso exame físico associado à uma radiografia da região torácica. Johnson et al. (2013) e Tobias \& Johnston (2013) mencionam também que, nestes exames, outros achados como enfisema subcutâneo e pneumotórax podem aparecer, como foi o caso do presente relato.

Não são todas as lesões torácicas que requerem intervenção cirúrgica, pois nem todas inviabilizam a função desta estrutura. Griffin \& Holt (2001) relatam que, aquelas que necessitam de procedimentos operatórios, geralmente são provenientes de lesões traumáticas penetrantes ou não. Estes ferimentos, juntamente com fraturas de costela, são frequentemente causados por mordidas de outros cães, acidentes automobilísticos e impactos de alta velocidade, como arma de fogo (Griffin \& Holt, 2001). No caso relatado, as lesões mencionadas foram causadas por mordida de um outro cão que, por sua extensão e gravidade, necessitaram de uma intervenção cirúrgica.

A técnica cirúrgica consistiu em acessar o local lesionado para excisar o tecido desvitalizado, inspecionar supostas lesões internas do pulmão, colocar uma sonda torácica para restabelecer a pressão intrapleural e fechar a cavidade, devolvendo a estabilidade dessa estrutura. Fossum (2014) afirma que é recomendada a exploração cirúrgica em casos de mordedura em região torácica com a finalidade de desbridar o tecido desvitalizado e reduzir o grau de contaminação.

No pós-operatório o paciente ficou sob cuidados intensivos, por um período de 72 horas, com o uso de antibiótico, anti-inflamatório com alta seletividade CoX-2, analgésico opioide e protetor gástrico. Johnson et al. (2013) e Tobias \& Johnston (2013) salientam que a terapia pós-operatória, consiste nestas medicações, dando uma atenção especial a analgesia para o controle da dor. Além disso, foi drenado o ar residual do tórax diariamente até o momento em que se obteve uma drenagem inferior a $20-30 \mathrm{ml} /$ conteúdo gasoso em 24 horas, sendo este o momento indicado por Aguiar (2011) para a retirada do dreno.

\section{Conclusão}

O respectivo relato demonstra a importância de um protocolo emergencial no atendimento de politraumatizados, trazendo agilidade diagnóstica e, consequente, direcionamento para o tratamento mais adequado e eficaz, neste caso cirúrgico, levando a um melhor prognóstico e sobrevida do paciente.

\section{Referências bibliográficas}

Aguiar, E. S. V. (2011). Emergências decorrentes do trauma em pequenos animais. Porto Alegre: Editora.

Bastos, R., Calhoon, J. H., \& Baisden, C. E. (2008). Flail chest and pulmonary contusion. Seminars in Thoracic and Cardiovascular Surgery, 20(1), 39-45. DOI: https://doi.org/10.1053/j.semtcvs.2008.01.004

Fossum, T. W. (2014). Cirurgia de pequenos animais (4th ed., Vol. 1). Elsevier Brasil.

Griffin, G. M., \& Holt, D. E. (2001). Dog-bite wounds: bacteriology and treatment outcome in 37 cases. Journal of the American Animal Hospital Association, 37(5), 453-460. DOI: 
https://doi.org/10.5326/15473317-37-5-453

Johnson, L. A., Orton, C. E., Willard, M. D., \& Carroll, C. L. (2013). Small animal surgery. Missouri: Elsevier Mosby.

Nelson, R. W., \& Couto, C. G. (2015). Medicina interna de pequenos animais (Issue 1). Elsevier Editora.

Olsen, D., Renberg, W., Perrett, J., Hauptman, J. G., Waldron, D. R., \& Monnet, E. (2002). Clinical management of flail chest in dogs and cats: a retrospective study of 24 cases (1989-1999). Journal of the American Animal Hospital Association, 38(4), 315-320. DOI: https://doi.org/10.5326/0380315

Slatter, D. H. (2007). Manual de cirurgia de pequenos animais. Manole.

Tobias, K. M., \& Johnston, S. A. (2013). Veterinary Surgery: Small Animal-E-BOOK: 2-Volume Set. Elsevier Health Sciences.

Truitt, M. S., Murry, J., Amos, J., Lorenzo, M., Mangram, A., Dunn, E., \& Moore, E. E. (2011). Continuous intercostal nerve blockade for rib fractures: ready for primetime? Journal of Trauma and Acute Care Surgery, 71(6), 1548-1552. DOI: https://doi.org/10.1097/ta.0b013e31823c96e0

Recebido: 30 de abril, 2020.

Aprovado: 9 de junho, 2020

Disponível online: 7 de outubro, 2020
Licenciamento: Este artigo é publicado na modalidade Acesso Aberto sob a licença Creative Commons Atribuição 4.0 (CC-BY 4.0), a qual permite uso irrestrito, distribuição, reprodução em qualquer meio, desde que o autor e a fonte sejam devidamente creditados. 